\title{
A Convenient Synthesis of Thiol, Trithiocarbonate and Disulfide
}

\author{
Sudershan R. Gondi*, Christiana Julia Rissing, and David Y. Son \\ Department of Chemistry, Southern Methodist University, Dallas, TX 75275-0314, U.S.A.
}

\begin{abstract}
Synthesis of unsymmetrical trithiocarbonate sulfonate salt, along with disulfide, thiol and symmetrical trithiocarbonate from 3-mercapto-1-propane-sulfonicacid, sodium salt with, without of phase transfer catalyst and under various reaction conditions are described. The obtained compounds having divergent usefulness in RAFT polymerization, sulfonyl preparation and having capable of binding in a multidentate fashion to soft transition metal ions.
\end{abstract}

\section{Keywords}

Symmetrical and unsymmetrical trithiocarbonate, disulfide, thiol, sulfonate salts.

\section{INTRODUCTION}

The industrial demand for novel synthetic materials with specific properties is constantly growing. From an academic point of view, this has resulted in tremendous effort being put into the research and development of new methods of polymerization that yield polymers with tailored structures and the desired properties. Preparation of polymers with controlled architectures can be achieved by making use of controlled polymerization ${ }^{1-2}$ techniques such as ionic polymerization, nitroxide-mediated polymerization (NMP), atom transfer radical polymerization (ATRP), and lately, (RAFT) reversible addition fragmentation chain transfer polymerization. ${ }^{3}$ Of all these methods, RAFT polymerization is often reported as being the most 
versatile, as it is fairly tolerant to impurities and can be used with a wide range of monomers. The control in RAFT-mediated polymerization is achieved by using trithiocarbonate compounds. ${ }^{4-6}$. Usually, thiol in presence of $\mathrm{CS}_{2}$ and halides are used for synthesis of trithiocarbonate..$^{2,7-9}$

\section{Present Work:}

This present article deals with the preparation of trithiocarbonate salt (1) from commercially available, water soluble, sodium salt of 3-mercapto-1-propane-sulfonic acid in presence of carbon disulfide and $\mathrm{NaOH}$, which is stable intermediate and stored in solution. The salt $\mathbf{1}$ reacts with bromo compounds $\mathbf{2}$ gives unsymmetrical $\mathbf{3}$ (organic salt). The stability of $\mathbf{3}$ depends on bromo substrate and reaction conditions. In presence of phase transfer catalyst 3 gives thiol (4), without PTC at room gives symmetrical trithiocarbonate (5), and water-soluble sulfonate polymer (6) and without PTC under heating conditions disulfide (7). (scheme-1).

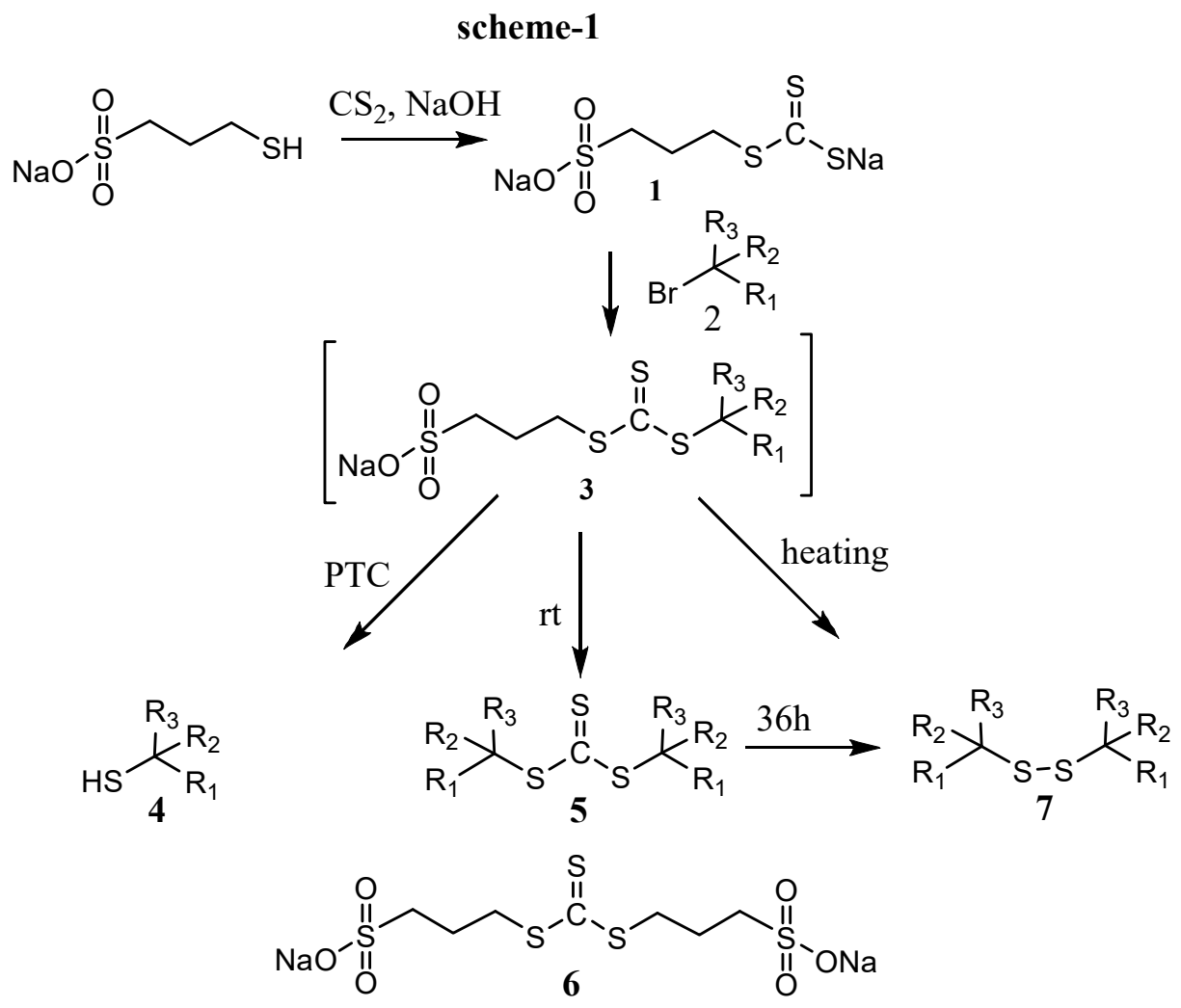


When intermediate, trithiocarbonate sodium salt (1) reacts with primary bromide (2a-2d), secondary bromide (2e-2f) and tertiary bromide (2g) in presence of catalytic amount of phase transfer catalyst, gives unsymmetrical salt (3), which subsequently cleaved to give thiol (4a-4g). When bromo compounds not having beta phenyl group (2h-2j) gives unsymmetrical trithiocarbonate salt (3h-3j). Under this condition, it is observed that nitrile (4d) was hydrolyzed to amide and the methyl ester (4f) was saponified to corresponding acid. (table-1).

Table-1, with PTC

\begin{tabular}{|ccccccccc|}
\hline entry & Substrate & $\mathbf{R}_{\mathbf{1}}$ & $\mathbf{R}_{\mathbf{2}}$ & $\mathbf{R}_{\mathbf{3}}$ & $\begin{array}{c}\text { unsym- } \\
\text { TTC (3) }\end{array}$ & yield & $\begin{array}{c}\text { Thiol } \\
\text { (4) }\end{array}$ & yield \\
\hline 1 & $2 \mathrm{a}$ & $\mathrm{MeOC}_{2} \mathrm{H}_{4} \mathrm{OCH}_{2}$ & $\mathrm{H}$ & $\mathrm{H}$ & & & $4 \mathrm{a}$ & 65 \\
2 & $2 \mathrm{~b}$ & $\mathrm{CH}_{2} \mathrm{COOH}$ & $\mathrm{H}$ & $\mathrm{H}$ & & & $4 \mathrm{~b}$ & 78 \\
3 & $2 \mathrm{c}$ & $\mathrm{COOH}$ & $\mathrm{H}$ & $\mathrm{H}$ & & & $4 \mathrm{c}$ & 72 \\
4 & $2 \mathrm{~d}$ & $\mathrm{CN}$ & $\mathrm{H}$ & $\mathrm{H}$ & & & $4 \mathrm{~d}$ & 72 \\
5 & $2 \mathrm{e}$ & $\mathrm{COOH}$ & $\mathrm{H}$ & $\mathrm{CH}_{3}$ & & & $4 \mathrm{e}$ & 73 \\
6 & $2 \mathrm{f}$ & $\mathrm{COOCH}$ & $\mathrm{H}$ & $\mathrm{CH}_{3}$ & & & $4 \mathrm{f}$ & 84 \\
7 & $2 \mathrm{~g}$ & $\mathrm{COOH}$ & $\mathrm{CH}_{3}$ & $\mathrm{CH}_{3}$ & & & $4 \mathrm{~g}$ & 63 \\
8 & $2 \mathrm{~h}$ & $\mathrm{Ph}$ & $\mathrm{H}$ & $\mathrm{CH}_{3}$ & $3 \mathrm{~g}$ & 95 & $4 \mathrm{~h}$ & 0 \\
9 & $2 \mathrm{i}$ & $4-\mathrm{Br}-\mathrm{Ph}$ & $\mathrm{H}$ & $\mathrm{H}$ & $3 \mathrm{~h}$ & 98 & $4 \mathrm{i}$ & 0 \\
10 & $2 \mathrm{~J}$ & $4-\mathrm{OMe}-\mathrm{Ph}$ & $\mathrm{H}$ & $\mathrm{H}$ & $3 \mathrm{j}$ & 95 & $4 \mathrm{j}$ & 0 \\
\hline
\end{tabular}

When intermediate trithiocarbonate sodium salt (1) reacts with substrate $(\mathbf{2 e}, \mathbf{2 g}-\mathbf{2 k})$ gives respective symmetrical trithiocarbonate $(\mathbf{5 e}, \mathbf{5} \mathbf{g}-\mathbf{5 k})$ along with sulfonate salt (6). The compound $\mathbf{6}$ is freely soluble in water, and its structure is confirmed by single crystal data. (table-2). 
Table-2, without PTC at room temperature

\begin{tabular}{|c|c|c|c|c|c|c|c|c|}
\hline entry & Substrate & $\mathbf{R}_{\mathbf{1}}$ & $\mathbf{R}_{\mathbf{2}}$ & $\mathbf{R}_{\mathbf{3}}$ & $\begin{array}{l}\text { sym- } \\
\text { TTC (5) } \\
\end{array}$ & yield & $\begin{array}{c}\text { Disulfide } \\
(7)\end{array}$ & yield \\
\hline 1 & $2 e$ & $\mathrm{COOH}$ & $\mathrm{H}$ & $\mathrm{CH}_{3}$ & $5 e$ & 91 & & \\
\hline 2 & $2 g$ & $\mathrm{COOH}$ & $\mathrm{CH}_{3}$ & $\mathrm{CH}_{3}$ & $5 i$ & 62 & & \\
\hline 3 & $2 \mathrm{~h}$ & $\mathrm{Ph}$ & $\mathrm{H}$ & $\mathrm{CH}_{3}$ & $5 \mathrm{~h}$ & 80 & & \\
\hline 4 & $2 i$ & 4-Br-Ph & $\mathrm{H}$ & $\mathrm{H}$ & $5 i$ & 70 & & \\
\hline 5 & $2 j$ & 4-OMe-Ph & $\mathrm{H}$ & $\mathrm{H}$ & $5 j$ & 78 & & \\
\hline 6 & $2 k$ & 4-Cl-Ph & $\mathrm{H}$ & $\mathrm{H}$ & $5 \mathrm{k}$ & 80 & & \\
\hline
\end{tabular}

When intermediate, trithiocarbonate sodium salt (1) reacts with primary bromide (2a), secondary bromide (2h-2k) under heating condition gives corresponding disulfide (7a, 7h-7k) at $\mathrm{pH} 14$ by extracting with organic solvents, upon acidification ( $\mathrm{pH} 4)$ of aqueous layer, respective thiols (4a, 4h-4k) were isolated (table-3). This method is superior for synthesis of disulfide in odor free environment, over its preparation from sulfide, ${ }^{10-11}$ halides, ${ }^{12-13}$ and metal sulfur. ${ }^{14}$ The PTC method as well as heating $\left(80^{\circ} \mathrm{C}\right)$ method are superior for thiol synthesis over reported methods from halide ${ }^{15-16}$ and in particular from thiourea ${ }^{17}$ under basic condition, and by reacting alcohol with Lawesson's reagent ${ }^{18-19}$.

Table-3, without PTC at heating

\begin{tabular}{|ccccccccc|}
\hline & & & & & Thiol & \multicolumn{2}{c|}{ Disulfide } \\
entry & Substrate & $\mathbf{R}_{\mathbf{1}}$ & $\mathbf{R}_{\mathbf{2}}$ & $\mathbf{R}_{\mathbf{3}}$ & $\mathbf{( 4 )}$ & yield & $\mathbf{( 7 )}$ & yield \\
\hline 1 & $2 \mathrm{a}$ & $\mathrm{MeOC}_{2} \mathrm{H}_{4} \mathrm{OCH}_{2}$ & $\mathrm{H}$ & $\mathrm{H}$ & $4 \mathrm{a}$ & 44 & $7 \mathrm{a}$ & 42 \\
2 & $2 \mathrm{~h}$ & $\mathrm{Ph}$ & $\mathrm{H}$ & $\mathrm{CH}_{3}$ & $4 \mathrm{~h}$ & 36 & $7 \mathrm{~h}$ & 40 \\
3 & $2 \mathrm{i}$ & $4-\mathrm{Br}-\mathrm{Ph}$ & $\mathrm{H}$ & $\mathrm{H}$ & $4 \mathrm{i}$ & 40 & $7 \mathrm{i}$ & 38 \\
4 & $2 \mathrm{j}$ & $4-\mathrm{OMe}-\mathrm{Ph}$ & $\mathrm{H}$ & $\mathrm{H}$ & $5 \mathrm{j}$ & 38 & $7 \mathrm{j}$ & 36 \\
5 & $2 \mathrm{k}$ & $4-\mathrm{Cl}-\mathrm{Ph}$ & $\mathrm{H}$ & $\mathrm{H}$ & $4 \mathrm{k}$ & 42 & $7 \mathrm{k}$ & 40 \\
\hline
\end{tabular}

\section{MECHANISM:}

PART-1: The unsymmetrical trithiocarbonate (3) in equilibrium as cyclic sulfone ${ }^{20}(\mathbf{C} 7)$ and thiolate ion (Ta). In presence of PTC catalyst, the equilibrium shift forwards, as primary bromide 
substrate (2a-2c), secondary bromide substrate (2d-2f) tertiary bromide substrate (2g) gives thiols, where sodium thiolate ion (Ta) gives thiols (4a-4e and $\mathbf{4 g}$ ) on acidification. In case of substrate with beta phenyl groups $(\mathbf{2} \mathbf{h}-\mathbf{2} \mathbf{j})$, the equilibrium is backward in undissociated stage (3h-3j) and not given any thiols. It is observed that PTC preventing the self-coupling of thiolate ion (Ta) to form disulfide (7) and preventing chain propagation reaction ie., attaching on another molecule of $\mathbf{3}$ in the formation of $\mathbf{6}$. (scheme-2)

PART-2: without PTC catalyst, the sodium thiolate ion (Ta) (initiation) attacks another molecule of $\mathbf{3}$ to give the symmetrical trithiocarbonate (TTC) 5 and 3-mercapto-1-propanesulfonicacid thiolate ion (Tb), as soon it forms, it will attack another molecule $\mathbf{3}$ to give the stable undissociated di sodium salt (6) by liberating sodium thiolate ion ( $\mathbf{T c})$, so that propagation of reaction goes on until consumption of $\mathbf{3}$. The aliquot workup shows symmetrical trithiocarbonate (3h and $\mathbf{3 i}$ ), which disappears after $12 \mathrm{~h}$ at room temperature. It is also confirmed that disulfide (DS) is not forming from trithiocarbonate (TTC) by doing independent reaction. (scheme-2). 


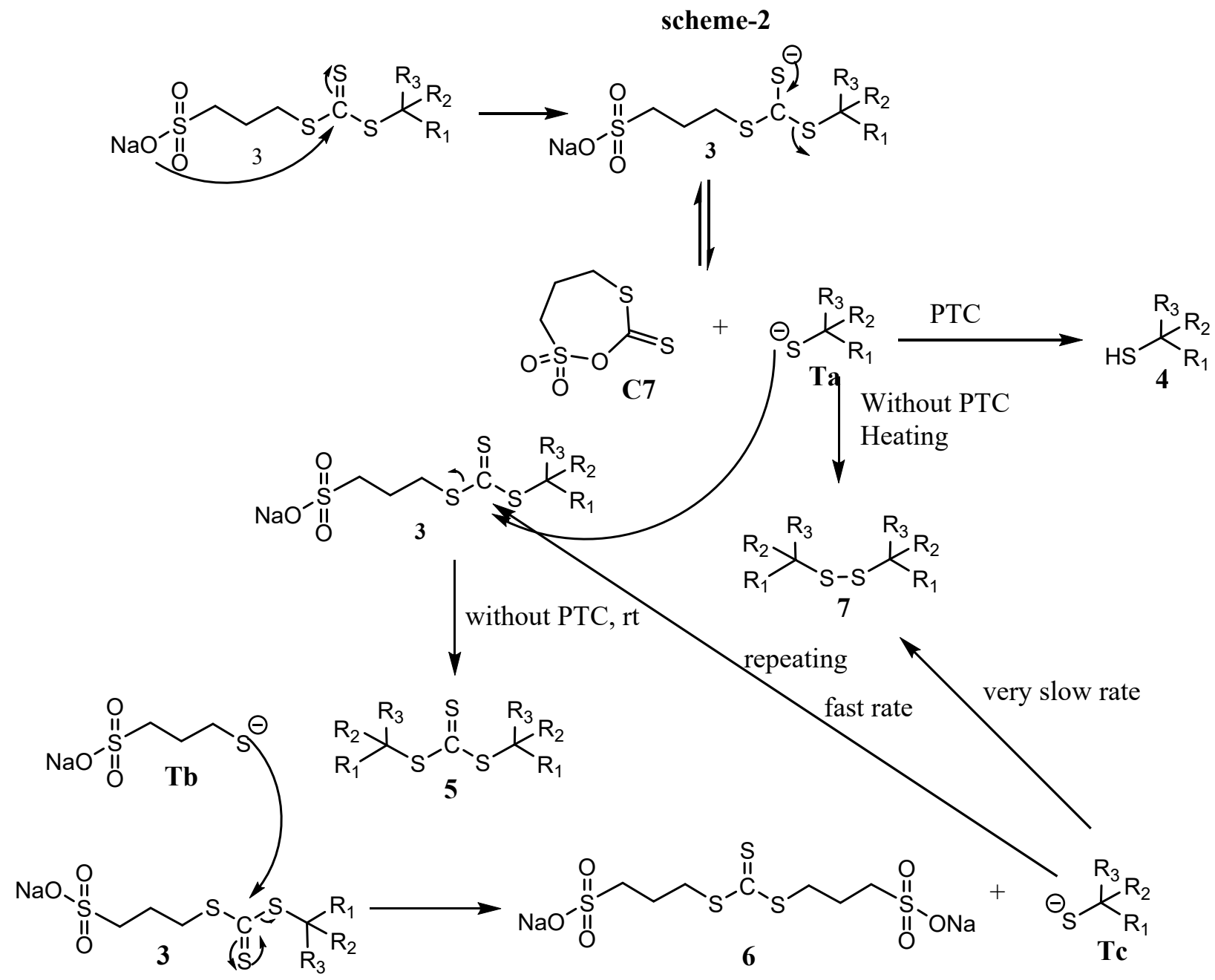

PART-3: without PTC and under heating condition $\left(80^{\circ} \mathrm{C}\right)$, thiol (4) formation takes place along with self-coupling of sodium thiolate ion (Ta) to gives the disulfide (7) in all cases. On prolonging the reaction time, exclusively disulfide product (7k) were isolated. Symmetrical trithiocarbonate (5) trace and disodium salt formation (6) is not detected under heating condition (scheme-2).

All new compounds were isolated as air and moisture-stable solids. All compounds were fully characterized using ${ }^{1} \mathrm{H}$ and ${ }^{13} \mathrm{C}$ NMR spectroscopy and elemental analysis. Organic salts are freely soluble in polar organic solvents DMSO and DMF and moderately soluble in methanol 
and ethanol, and insoluble in most organic solvents. The compound $\mathbf{6}$ is freely soluble in sat. $\mathrm{NaHCO}_{3}, \mathrm{pH}=9,10 \% \mathrm{HCl}$, water and dmso. Sodium salt of Trithiocarbonate (3) can be useful for transfer surfactants in emulsion polymerizations and symmetrical trithiocarbonate (5) for sulfonyl chloride synthesis ${ }^{21}$. These compounds (5 and 7) possess multiple sulfur atoms and are thus capable of binding in a multidentate fashion to soft transition metal ions. ${ }^{22-24}$ A reaction of these ligands with late transition metal ions is a current focus in our laboratory.

\section{Conclusions}

We have prepared a series of new unsymmetrical and symmetrical trithiocarbonate, thiol and disulfide compounds containing different other functional groups by using of commercially available, water soluble, mercaptan salt. The synthetic procedure is straightforward, and the products are obtained in good to excellent yields without any chromatographic purification. Excellent solubility properties and the presence of electron donor groups on arms of trithiocarbonate described herein may be advantageous in applications RAFT polymerization and ligand preparation using transition metals.

\section{ACKNOWLEDGMENTS}

The authors thank the National Science Foundation and the Welch Foundation for support of this work.

Declaration of Competing Interest The authors declare that they have no known competing financial interests.

Data and materials availability: Requests for materials should be addressed to Sudershan Gondi (gondisr@gmail.com) 


\section{EXPERIMENTAL SECTION:}

3-mercapto-1-propane-sulfonicacid, sodium salt and carbon disulfide were obtained from Acros and Aldrich respectively. All other materials were reagent grade unless otherwise specified. All reactions were carried out in a dry nitrogen atmosphere. 1H and 13C NMR spectra were obtained on a 400-MHz Bruker Avance NMR spectrometer. Infrared spectra were obtained on a Nicolet Magna-IR 560 spectrometer E.S.P. Elemental analyses were obtained with a CE Elantech Thermo-Finnigan Flash $1112 \mathrm{CHN}$ elemental analyzer. Melting points were collected on a TA Instruments DSC 2010 Differential Scanning Calorimeter using a heating rate of $108^{\circ} \mathrm{C} / \mathrm{min}$ and nitrogen as a purge gas.

3-Dithiocarboxysulfanyl-propane-1-sulfonicacid disodium salt (1): To a solution of 3-

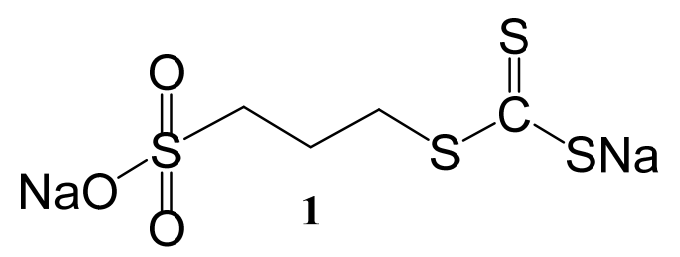
mercapto-1-propane-sulfonicacid, sodium salt, $(10.0 \mathrm{~g}$, $56.0 \mathrm{mmol})$ in $80 \mathrm{~mL}$ of water, add freshly prepared $(20 \mathrm{~mL})$ of $(6.73 \mathrm{~g}, 168 \mathrm{mmol}) \mathrm{NaOH}$ solution. After stirring at room temperature for 30 minutes, add carbon disulfide $(3.36 \mathrm{~mL}, 5.6 \mathrm{mmol})$ drop wise over a period of 5 minutes. Stir the reaction mixture for overnight $(18 \mathrm{~h})$. This is stock solution, which is stable for over months, $15.5 \mathrm{~g}$ (56 $\mathrm{mmol})$ in $100 \mathrm{~mL} .1 .56 \mathrm{~g}(5.6 \mathrm{mmol})$ of in situ salt in $10 \mathrm{~mL}$ or $1.0 \mathrm{~g}(3.6 \mathrm{mmol})$ of in situ salt in $6.45 \mathrm{~mL} .{ }^{1} \mathrm{H}-\mathrm{NMR}\left(400 \mathrm{MHz}, \mathrm{d}_{2} \mathrm{o}\right):{ }^{\delta} 3.30-3.26\left(\mathrm{t}, 2 \mathrm{H}, J=7.0 \mathrm{~Hz},-\mathrm{CH}_{2} \mathrm{SO}_{3}\right), 3.01-2.97(\mathrm{t}, 2 \mathrm{H}, J$ $\left.=7.2 \mathrm{~Hz},-\mathrm{CH}_{2} \mathrm{~S}\right), 2.10-2.07\left(\mathrm{~m}, 2 \mathrm{H},-\mathrm{CH}_{2} \mathrm{CH}_{2} \mathrm{~S}\right) .{ }^{13} \mathrm{C}-\mathrm{NMR}\left(100.6 \mathrm{MHz}, \mathrm{d}_{2} \mathrm{o}\right):{ }^{\delta} 50.4(-$ $\left.\mathrm{CH}_{2} \mathrm{SO}_{3}\right), 40.0\left(-\mathrm{CH}_{2} \mathrm{~S}\right), 23.9\left(-\mathrm{CH}_{2} \mathrm{CH}_{2} \mathrm{~S}\right)$. IR (KBr) (wavenumber, $\left.\mathrm{cm}^{-1}\right)$ : 3442, 2985 (C-Cs), 1638, 1443, 1408, 1194, $1048(\mathrm{C}=\mathrm{S}), 870(\mathrm{C}-\mathrm{Cb})$. Elemental Analysis, Calcd for $\mathrm{C}_{4} \mathrm{H}_{6} \mathrm{Na}_{2} \mathrm{O}_{3} \mathrm{~S}_{4}$ : C) $17.39 \%$, H) $2.19 \%$. Found: C) $17.39 \%$, H) $2.19 \%$. 
3-(1-Phenyl-ethylsulfanylthiocarbonylsulfanyl)-propane-1-sulfonic acid sodium salt ${ }^{25}$ (3h):

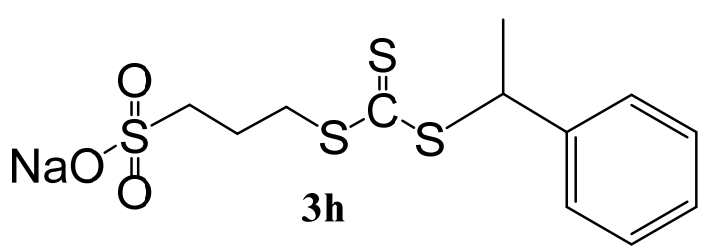

To a $6.45 \mathrm{~mL}(1.0 \mathrm{~g}, 3.6 \mathrm{mmol})$ in situ solution of in

3-dithiocarboxysulfanyl-propane-1-sulfonic acid disodium salt was added 1-bromoethyl benzene

(667 mg, $5.6 \mathrm{mmol})$, in presence of catalytic amount of tetrabutylammonium bromide $(20 \mathrm{mg})$.

Stir the reaction mixture for overnight $(12 \mathrm{~h})$, during reaction time yellow solid is formed. Dilute the reaction mass with water $(50 \mathrm{~mL})$, filter, washed with water $(50 \mathrm{~mL})$, ether $(50 \mathrm{~mL})$ and dry in vacuum to compound $\mathbf{4 g}$ as yellow solid (1.25 g, 98\%). Mp 282.6 ${ }^{\circ} \mathrm{C} .{ }^{1} \mathrm{H}-\mathrm{NMR}(400 \mathrm{MHz}$, dmso-d $\left._{6}\right):{ }^{\delta} 7.44-7.42(\mathrm{~d}, 2 \mathrm{H}, J=7.2 \mathrm{~Hz}, 2,6 \mathrm{Ar}-\boldsymbol{H}), 7.37-7.33(\mathrm{t}, 2 \mathrm{H}, J=7.0 \mathrm{~Hz}, 3,5 \mathrm{Ar}-\boldsymbol{H})$, 7.30-7.27 (t, 1H, $J=7.0 \mathrm{~Hz}, 4-\mathrm{Ar} \boldsymbol{H}), 5.30-5.24\left(\mathrm{q}, 1 \mathrm{H}, J=6.8 \mathrm{~Hz}, \mathrm{ArCHCH}_{3}\right), 3.48-3.44$ (t, 2H, $\left.J=7.1 \mathrm{~Hz},-\mathrm{CH}_{2} \mathrm{SO}_{3}\right), 2.50-2.47\left(\mathrm{t}, 2 \mathrm{H}, J=7.0 \mathrm{~Hz},-C \boldsymbol{H}_{2} \mathrm{~S}\right), 1.96-1.88\left(\mathrm{~m}, 2 \mathrm{H},-\mathrm{CH}_{2} \mathrm{CH}_{2} \mathrm{~S}\right) 1.71-$

$1.70(\mathrm{~d}, 3 \mathrm{H}, J=7.0 \mathrm{~Hz}, \operatorname{ArCHCH} 3) .{ }^{13} \mathrm{C}-\mathrm{NMR}(100.6 \mathrm{MHz}, \mathrm{dmso}-\mathrm{d} 6):{ }^{\delta} 141.6$ (C1-Ar), 129.5 (C2 \& C6-Ar), 128.6 (C3 \& C5-Ar), 128.4 (C4-Ar), $50.6\left(-\mathrm{CH}_{2} \mathrm{SO}_{3}\right), 50.6\left(\mathrm{Ar}-\mathrm{CH}-\mathrm{CH}_{3}\right), 36.2$ ($\left.\mathrm{CH}_{2} \mathrm{~S}\right), 24.9\left(-\mathrm{CH}_{2} \mathrm{CH}_{2} \mathrm{~S}\right), 22.0\left(\mathrm{Ar}-\mathrm{CH}_{-} \mathrm{CH}_{3}\right)$. IR (KBr) (wavenumber, $\left.\mathrm{cm}^{-1}\right)$ : 3509, 2929 (CCs), 1627, 1439, 1173, $1057(\mathrm{C}=\mathrm{S}), 820(\mathrm{C}-\mathrm{Cb})$. Elemental Analysis, Calcd for $\mathrm{C}_{12} \mathrm{H}_{15} \mathrm{NaO}_{3} \mathrm{~S}_{4}$ : C) $40.97 \%$, H) $4.38 \%$. Found: C) $40.27 \%$, H) $4.68 \%$.

\section{3-(4-Bromo-benzylsulfanylthiocarbonylsulfanyl)-propane-1-sulfonic acid sodium salt ${ }^{26}$ (3i):}<smiles>O=S(=O)(CCCSC(=S)SCc1ccc(Br)cc1)O[Na]</smiles>

In the manner described above, $6.45 \mathrm{~mL}$ (1.0 g, $3.6 \mathrm{mmol})$ in situ solution of 3 dithiocarboxysulfanyl-propane-1 sulfonic acid disodium salt was treated with 4-

bromo benzyl bromide $(0.905 \mathrm{mg}, 3.6 \mathrm{mmol})$ in presence catalytic amount of 
tetrabutylammonium bromide $(20 \mathrm{mg})$ to obtain compound $\mathbf{3 h}(1.45 \mathrm{~g}, 95 \%)$. MP $236.6^{\circ} \mathrm{C},{ }^{1} \mathrm{H}-$ NMR (400 MHz, dmso-d6): ${ }^{\delta} 7.51$ (s, 2H), $7.35(2 \mathrm{H}), 4.64(\mathrm{~s}, 2 \mathrm{H}), 3.49(\mathrm{~s}, 2 \mathrm{H}), 2.51(\mathrm{~s}, 2 \mathrm{H})$, 1.98 (s, 2H). ${ }^{13} \mathrm{C}-\mathrm{NMR}\left(400 \mathrm{MHz}, \mathrm{dmso-d}\right.$ ): ${ }^{\delta} 135.8,132.3,132.2,121.8,50.6,40.2,36.5,24.9$ IR (KBr): 3508, 2930, 1630,736 (medium), 1485, 1217, 1176, 1058, $820 \mathrm{~cm}^{-1}$ (strong). Elemental Analysis: Calcd: C, 31.21, H, 2.86. Found: C, 30.58, H, 3.26.

After acidification, Sulfonic acid shows $\mathrm{Mp} 240.82^{\circ} \mathrm{C} .{ }^{1} \mathrm{H}-\mathrm{NMR}\left(400 \mathrm{MHz}, \mathrm{dmso-d}\right.$ ): ${ }^{\delta} 7.53-$ NaO $\int_{1}^{-1}$ 1.96-1.94 (d, 2H, $\left.J=6.65 \mathrm{~Hz},-\mathrm{CH}_{2} \mathrm{CH}_{2} \mathrm{~S}\right) .{ }^{13} \mathrm{C}-\mathrm{NMR}\left(100.6 \mathrm{MHz}, \mathrm{dmso-d}\right.$ ): ${ }^{\delta} 135.8$ (C1-Ar), 132.3 (C2 \& C6-Ar), 132.2 (C3 \& C5-Ar), 121.6 (C4-Ar), $50.7\left(-\mathrm{CH}_{2} \mathrm{SO}_{3}\right), 40.2\left(\mathrm{Ar}-\mathrm{CH}_{2} \mathrm{~S}\right)$, $36.6\left(-\mathrm{CH}_{2} \mathrm{~S}\right), 24.9 \quad\left(-\mathrm{CH}_{2} \mathrm{CH}_{2} \mathrm{~S}\right) . \mathrm{IR}(\mathrm{KBr})$ (wavenumber, $\left.\mathrm{cm}^{-1}\right)$ : 3513, 2931 (C-Cs), 1627, 1486, 1287, 1173, $1064(\mathrm{C}=\mathrm{S}), 817(\mathrm{C}-\mathrm{Cb})$. Elemental Analysis, Calcd for $\left.\mathrm{C}_{11} \mathrm{H}_{13} \mathrm{BrO}_{3} \mathrm{~S}_{4}: \mathrm{C}\right)$ $31.21 \%$, H) $2.86 \%$. Found: C) $31.28 \%$, H) $3.17 \%$.

3-(4-methoxy-benzylsulfanylthiocarbonylsulfanyl)-propane-1-sulfonic acid sodium salt ${ }^{26}$<smiles>COc1ccc(CSC(=S)SCCCS(=O)(=O)O[Na])cc1</smiles>
(3j): In the manner described above, $6.45 \mathrm{~mL}(1.0 \mathrm{~g}, 3.6 \mathrm{mmol})$ in situ solution of 3-dithiocarboxysulfanylpropane-1 -sulfonic acid disodium salt was treated with 4-methoxy benzyl chloride $(0.562 \mathrm{mg}$, $3.6 \mathrm{mmol})$ in presence catalytic amount of tetrabutylammonium bromide $(20 \mathrm{mg})$ to obtain compound 3j (1.3 g, 95\%), ${ }^{1} \mathrm{H}-\mathrm{NMR}\left(400 \mathrm{MHz}, \mathrm{dmso}_{\mathrm{d}}\right):^{\delta} 7.30(\mathrm{~d}, J=8.4 \mathrm{~Hz}, 2 \mathrm{H}), 6.78$ (d, $J$ $=8.4 \mathrm{~Hz}, 2 \mathrm{H}), 4.59(\mathrm{~s}, 2 \mathrm{H}), 3.71(\mathrm{~s}, 3 \mathrm{H}), 3.47(\mathrm{t}, J=7.3 \mathrm{~Hz}, 2 \mathrm{H}), 2.58-243(\mathrm{~m}, 2 \mathrm{H}), 1.93(\mathrm{t}, J=$ 
$7.3 \mathrm{~Hz}, 2 \mathrm{H}),{ }^{13} \mathrm{C}-\mathrm{NMR}\left(400 \mathrm{MHz}, \mathrm{dmso}_{\mathrm{d}}\right):{ }^{\delta} 224.2,159.2,130,9,127.0,114.4,55.5,50.3$, $40.5,35.9,24.6$.

bis[2-(2-methoxyethoxy)-ethanethiol ${ }^{27}$ (4a): To a $6.45 \mathrm{~mL}(1.0 \mathrm{~g}, 3.6 \mathrm{mmol})$ in situ solution of

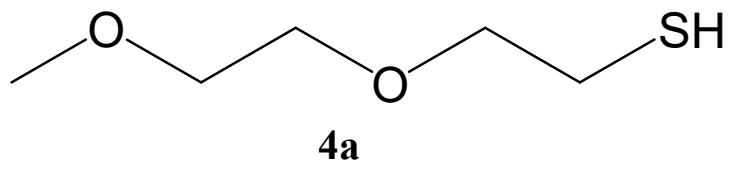
in 3-dithiocarboxysulfanyl-propane-1-sulfonicacid disodium salt was added 1-bromo-2-(2methoxyethoxy)-ethane, $(0.66 \mathrm{~g}, 3.6 \mathrm{mmol})$ in

presence of catalytic amount of tetra butyl ammonium bromide $(20 \mathrm{mg})$. Stir the reaction mixture for overnight $(12 \mathrm{~h})$. Dilute the reaction mixture with water $(50 \mathrm{~mL})$ and extract with ether $(50$ $\mathrm{mL}$ ). to remove the impurities. The aqueous layer on acidified with concentrated $\mathrm{HCl}$ to $\mathrm{pH}=2$,

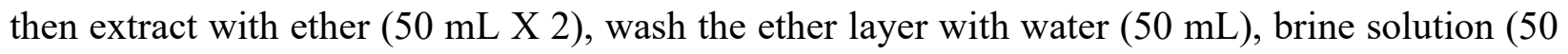
$\mathrm{mL}$ ), dried over $\mathrm{MgSO}_{4}$ and filter. The filtrate was concentrated to give thiol as residual oil. (640 $\mathrm{mg}, 65 \%) .{ }^{1} \mathrm{H}-\mathrm{NMR}\left(400 \mathrm{MHz}, \mathrm{cdcl}_{3}\right):{ }^{8} 3.63 .-3.60$ (t, $4 \mathrm{H}, \mathrm{J}=6.1 \mathrm{~Hz}, \mathrm{CH}_{3} \mathrm{O}-\mathrm{CH}_{2}-\mathrm{CH}_{2} \mathrm{O}-$ ), 3.56$3.54\left(\mathrm{t}, 2 \mathrm{H}, J=4.5 \mathrm{~Hz},-\mathrm{OCH}_{2} \mathrm{CH}_{2} \mathrm{~S}\right), 3.38$ (s, $\left.3 \mathrm{H},-\mathrm{OCH}_{3}\right), 2.73-2.68\left(\mathrm{~m}, 2 \mathrm{H},-\mathrm{CH}_{2} \mathrm{~S}\right), 1.61-1.57$ (t, $\left.1 \mathrm{H}, \mathrm{SH}, J=8.2 \mathrm{~Hz},-\mathrm{CH}_{2} \mathrm{~S}\right) \cdot{ }^{13} \mathrm{C}-\mathrm{NMR}\left(100.6 \mathrm{MHz}, \mathrm{cdcl}_{3}\right):{ }^{\delta} 71.3\left(\mathrm{CH}_{3} \mathrm{O}-\mathrm{CH}_{2}-\mathrm{CH}_{2} \mathrm{O}-\right), 70.6$ $\left(\mathrm{CH}_{3} \mathrm{O}-\mathrm{CH}_{2}-\mathrm{CH}_{2} \mathrm{O}-\right), 69.8\left(-\mathrm{OCH}_{2} \mathrm{CH}_{2} \mathrm{~S}\right), 58.4\left(-\mathrm{OCH}_{3}\right), 29.7\left(-\mathrm{CH}_{2} \mathrm{~S}\right)$. IR (KBr) (wavenumber, $\left.\mathrm{cm}^{-1}\right): 2877$ (C-Cs), 1454, 1355, 1293, 1198, $1140(\mathrm{C}=\mathrm{S}), 735(\mathrm{C}-\mathrm{Cb})$. Elemental Analysis, Calcd for $\mathrm{C}_{5} \mathrm{H}_{12} \mathrm{O}_{2} \mathrm{~S}:$ C) $44.09 \%$, H) 8.88\%. Found: C) 44.01\%, H) $9.19 \%$.

3-Mercaptopropanoic $\mathbf{a c i d}^{\mathbf{2 8}}$ (4b): In the manner described above, To a $6.45 \mathrm{~mL}(1.0 \mathrm{~g}, 3.6$ HS mmol) in situ solution of 3-dithiocarboxysulfanyl-propane-1-sulfonicacid disodium salt was treated 3-bromopropanoicacid, $(0.55 \mathrm{~g}, 3.6 \mathrm{mmol})$ in presence catalytic amount of tetrabutylammonium bromide $(20 \mathrm{mg})$ to obtain product as residual oil (296 mg, 77.8\%). ${ }^{1} \mathrm{H}-\mathrm{NMR}\left(400 \mathrm{MHz}, \mathrm{CDCl}_{3}\right):{ }^{\delta} 11.67$ (br-s, $1 \mathrm{H}$, $\mathrm{COOH}$ ), 2.77-267 (m, 4H, $\mathrm{HSCH}_{2} \mathrm{CH}_{2}$ ), 1.69-1.67 (t, $\left.J=7.8 \mathrm{~Hz}, \mathrm{SH}\right)$. NMR (400 MHz, d20): ${ }^{\delta}$ 
2.657-261 (m, 4H, $\left.\mathrm{HSCH}_{2} \mathrm{CH}_{2}\right),{ }^{13} \mathrm{C}-\mathrm{NMR}\left(100.6 \mathrm{MHz}, \mathrm{CDCl}_{3}\right):{ }^{\delta} 178.0(\mathbf{C O O H}), 38.1$ $\left.\left(\mathrm{HSCH}_{2} \mathrm{CH}_{2}\right), 19.1\left(\mathrm{HOOCCH}_{2} \mathrm{CH}_{2}\right) .{ }^{13} \mathrm{C}-\mathrm{NMR}\left(100.6 \mathrm{MHz}, \mathrm{d}_{2}\right):\right):{ }^{\delta} 178.6(\mathrm{COOH}), 38.2$ $\left(\mathrm{HSCH}_{2} \mathrm{CH}_{2}\right), 19.3 \mathrm{HOOCCH}_{2} \mathrm{CH}_{2}$ ).

2-Mercaptoacetic acid ${ }^{29}(4 \mathrm{c})$ : In the manner described above, To a $6.45 \mathrm{~mL}(1.0 \mathrm{~g}, 3.6 \mathrm{mmol})$ in situ solution of 3-dithiocarboxysulfanyl-propane-1-sulfonicacid disodium salt was treated 2-bromo acetic acid, $(0.5 \mathrm{~g}, 3.6 \mathrm{mmol})$ in presence catalytic residual oil (235 mg,71.6\%). ${ }^{1} \mathrm{H}-\mathrm{NMR}\left(400 \mathrm{MHz}, \mathrm{CDCl}_{3}\right):{ }^{8} 3.06$ (s, $\left.2 \mathrm{H}, \mathrm{HSCH}_{2} \mathrm{COOH}\right),{ }^{13} \mathrm{C}-$ NMR (100.6 MHz, d $\left.\mathrm{d}_{20}\right){ }^{\delta} 178.6(\mathrm{COOH}), 29.0\left(\mathrm{HSCH}_{2} \mathrm{COOH}\right)$,

2-Mercapto-2-methyl-propanamide ${ }^{\mathbf{3 0}}$ (4d): In the manner described above, To a $6.45 \mathrm{~mL}(1.0$

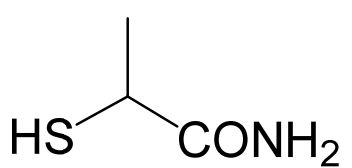

$4 d$ $\mathrm{g}, 3.6 \mathrm{mmol}$ ) in situ solution of 3-dithiocarboxysulfanyl-propane-1sulfonicacid disodium salt was treated 2-bromo propionitrile, $(0.48 \mathrm{~g}, 3.6$ $\mathrm{mmol})$ in presence catalytic amount of tetrabutylammonium bromide (20 $\left.\left.1 \mathrm{H},-\mathrm{CHCH}_{3}\right), 1.46-1.51\left(\mathrm{~m}, 3 \mathrm{H},-\mathrm{CHCH}_{3}\right) .{ }^{13} \mathrm{C}-\mathrm{NMR}\left(100.6 \mathrm{MHz}, \mathrm{d}_{20}\right):\right):{ }^{\delta} 178.2\left(\mathrm{CONH}_{2}\right)$, $42.9\left(-\mathrm{CHCH}_{3}\right), 17.6\left(-\mathrm{CHCH}_{3}\right)$.

2-Mercapto-propanoic acid ${ }^{31}$ (4e): To a $6.45 \mathrm{~mL}(1.0 \mathrm{~g}, 3.6 \mathrm{mmol})$ situ solution of in 3-<smiles>CC(S)C(=O)O</smiles>

$4 e$ dithiocarboxysulfanyl-propane-1-sulfonicacid disodium salt was added 2bromopropanoicacid, (560 mg, $3.6 \mathrm{mmol})$ in presence of catalytic amount of tetra butyl ammonium bromide $(20 \mathrm{mg})$. Stir the reaction mixture for overnight $(12 \mathrm{~h})$. Dilute the reaction mixture with water $(50 \mathrm{~mL})$ and extract with ether $(50 \mathrm{~mL})$ to discard the impurities. The aqueous layer on acidified with concentrated $\mathrm{HCl}$ to $\mathrm{pH}=2$, then extract with ether $(50 \mathrm{~mL} \mathrm{X} \mathrm{2)}$, and the organic layer was washed with water $(100 \mathrm{~mL})$, brine 
$(100 \mathrm{~mL})$, and then dried over anhydrous magnesium sulfate and filtered. The filtrate was concentrated to give product as residual oil (280 mg, 73\%). ${ }^{1} \mathrm{H}-\mathrm{NMR}\left(400 \mathrm{MHz}, \mathrm{CDCl}_{3}\right):{ }^{\delta} 11.14$ (br-s, $1 \mathrm{H}, \mathrm{COOH}), 3.58-3.50\left(\mathrm{~m}, 1 \mathrm{H}, \mathrm{HSCHCH}_{3}\right), 2.25-2.23(\mathrm{~s}, J=7.8 \mathrm{~Hz}, 1 \mathrm{H},-\mathrm{SH}) 1.55-1.53$ $(\mathrm{d}, \quad J=7.8 \mathrm{~Hz}, \mathrm{HOOCCHCH} 3) .{ }^{13} \mathrm{C}-\mathrm{NMR}\left(100.6 \mathrm{MHz}, \mathrm{d}_{2 \mathrm{O}}\right):{ }^{\delta} 180.0(\boldsymbol{C O O H}), 35.5$ $\left(\mathrm{HSCHCH}_{3}\right), 20.6\left(\mathrm{HOOCCHCH}_{3}\right)$.

2-Mercapto-propanoic acid $^{\mathbf{3 1}}$ (4e): In the manner described above, To a $6.45 \mathrm{~mL}(1.0 \mathrm{~g}, 3.6$

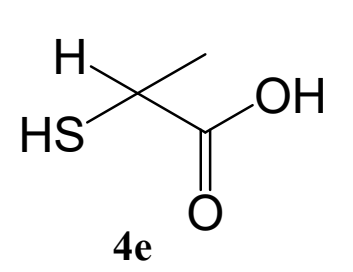
mmol) in situ solution of 3-dithiocarboxysulfanyl-propane-1-sulfonic acid di sodium salt was treated methyl, 2-bromopropionoate, $(0.55 \mathrm{~g}, 3.6 \mathrm{mmol})$ in presence catalytic amount of tetrabutylammonium bromide $(20 \mathrm{mg})$ to obtain product as residual oil $(327 \mathrm{mg}, 84 \%)$. NMR values are consistent with the reported values.

2-Mercapto-2-methyl-propanoic $\operatorname{acid}^{\mathbf{3 2}}$ (4g): In the manner described above, To a $6.45 \mathrm{~mL}$

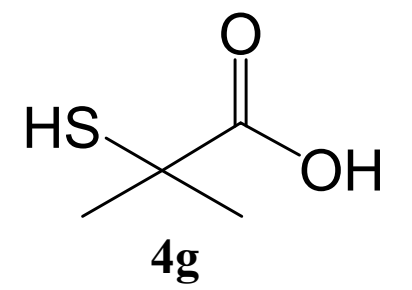
$(1.0 \mathrm{~g}, 3.6 \mathrm{mmol})$ in situ solution of 3-dithiocarboxysulfanyl-propane1-sulfonicacid disodium salt was treated 2-bromo-2-methyl propanoic acid, $(0.6 \mathrm{~g}, \quad 3.6 \mathrm{mmol})$ in presence catalytic amount of tetrabutylammonium bromide $(20 \mathrm{mg})$ to obtain product as solid. $(270$ mg, 63\%). Mp 194-196 C. ${ }^{1} \mathrm{H}-\mathrm{NMR}\left(400 \mathrm{MHz}, \mathrm{dmso}-\mathrm{d}\right.$ ): ${ }^{\delta} 12.74$ (br-s, 1H, COOH), 3.45 (s, 1H, HS), $1.44\left(\mathrm{~s}, 6 \mathrm{H}, \mathrm{HSC}\left(\mathrm{CH}_{3}\right)_{2}\right){ }^{13} \mathrm{C}-\mathrm{NMR}\left(100.6 \mathrm{MHz}, \mathrm{d}_{20}\right):{ }^{\delta} 180.0$ (COOH), 57.5 $\left.\left(\mathrm{HSCHCH}_{3}\right), 30.7\left(\mathrm{HOOCCCH}_{3}\right)_{2}\right)$. IR (KBr) (wavenumber, $\left.\mathrm{cm}^{-1}\right)$ : 298010, 2654 (C-Cs), 1688, 1464, 1289, 1165, $1113(\mathrm{C}=\mathrm{S}), 809(\mathrm{C}-\mathrm{Cb})$.

3-(3-Sulfo-propylsulfanylthiocarbonylsulfanyl)-propane-1-sulfonic acid, di sodium salt (6):

To a $6.45 \mathrm{~mL}(1.0 \mathrm{~g}, 3.6 \mathrm{mmol})$ in situ solution of in<smiles>[NH3+]OS(=O)(=O)CCCSC(=S)SCCCS(=O)(=O)O[Na]</smiles>

3-dithiocarboxysulfanyl-propane-1-sulfonicacid

Page 13 of 23 
disodium salt was added 2-bromo propanoic acid, $(430 \mathrm{mg}, 3.6 \mathrm{mmol})$. Stir the reaction mixture for overnight $(12 \mathrm{~h})$, during reaction time yellow solid is formed. Filter the yellow solid, suspended in acetone, filter and dry in vacuum to give compound 6 (550 $\mathrm{mg}, 44.1 \%)$. Mp $>250^{\circ} \mathrm{C} .{ }^{1} \mathrm{H}-\mathrm{NMR}\left(400 \mathrm{MHz}, \mathrm{dmso}_{6}\right.$ ): ${ }^{\delta} 3.50-3.47$ (t, 4H, $\left.J=6.8 \mathrm{~Hz},-\mathrm{CH}_{2} \mathrm{SO}_{3}\right), 2.55-2.51(\mathrm{t}$, $\left.4 \mathrm{H}, J=7.2 \mathrm{~Hz},-\mathrm{CH}_{2} \mathrm{~S}\right), 1.96-1.93\left(\mathrm{t}, 4 \mathrm{H}, J=7.0 \mathrm{~Hz},-\mathrm{CH}_{2} \mathrm{CH}_{2} \mathrm{~S}\right) .{ }^{13} \mathrm{C}-\mathrm{NMR}(100.6 \mathrm{MHz}$, dmsod6): ${ }^{\delta} 50.6\left(-\mathrm{CH}_{2} \mathrm{SO}_{3}\right), 36.2\left(-\mathrm{CH}_{2} \mathrm{~S}\right), 24.9\left(-\mathrm{CH}_{2} \mathrm{CH}_{2} \mathrm{~S}\right) .{ }^{1} \mathrm{H}-\mathrm{NMR}\left(400 \mathrm{MHz}, \mathrm{d}_{2} \mathrm{o}\right):{ }^{\delta} 3.50-3.47$ (t, $\left.2 \mathrm{H}, J=7.2 \mathrm{~Hz},-\mathrm{CH}_{2} \mathrm{SO}_{3}\right), 2.96-2.92\left(\mathrm{t}, 2 \mathrm{H}, J=7.2 \mathrm{~Hz},-\mathrm{CH}_{2} \mathrm{~S}\right), 2.13-2.05(\mathrm{~m}, 2 \mathrm{H},-$ $\left.\mathrm{CH}_{2} \mathrm{CH}_{2} \mathrm{~S}\right) .{ }^{13} \mathrm{C}-\mathrm{NMR}\left(100.6 \mathrm{MHz}, \mathrm{d}_{2} \mathrm{o}\right):{ }^{\delta} 50.0\left(-\mathrm{CH}_{2} \mathrm{SO}_{3}\right), 35.2\left(-\mathrm{CH}_{2} \mathrm{~S}\right), 23.6\left(-\mathrm{CH}_{2} \mathrm{CH}_{2} \mathrm{~S}\right)$. IR (KBr) (wavenumber, $\left.\mathrm{cm}^{-1}\right)$ : 3510, $2931(\mathrm{C}-\mathrm{Cs}), 1626,1418,1216,1172,1049$ (C=S), $828(\mathrm{C}-$ $\mathrm{Cb}$ ). Elemental Analysis, Calcd for $\mathrm{C}_{7} \mathrm{H}_{12} \mathrm{BrNa}_{2} \mathrm{O}_{6} \mathrm{~S}_{5}:$ C) $21.10 \%$, H) 3.05\%. Found: C) $21.14 \%$, H) $3.70 \%$. Single crystal data confirmed the structure.

2-mercapto propanoic acid trithiocarbonate ${ }^{33}(5 e)$ : The aqueous layer on acidified with<smiles>CC(SC(=S)SC(C)C(=O)O)C(=O)O</smiles>
concentrated $\mathrm{HCl}$ to $\mathrm{pH}$ 2, Filter the yellow solid and dry in vacuum to give trithiocarbonate $\mathbf{5 e}(410 \mathrm{mg}$, 91\%). NMR values are consistent with the reported values.

2,2'-[(Thioxomethylene)disulfanyl]bis(2-methylpropanoic acid) $)^{7} \mathbf{( 5 g )}$ : In the manner<smiles>CC(C)(SC(=S)SC(C)(C)C(=O)O)C(=O)O</smiles>
described above, $2.5 \mathrm{~g}$ (14 mmol) of 3-mercapto-1propane-sulfonicacid sodium salt was treated with sodium hydroxide (1.68 g, $42 \mathrm{mmol})$, carbon disulfide (1.06 g, $0.84 \mathrm{~mL}, 14 \mathrm{mmol})$ followed by 2-bromo-2-methyl-propanoic acid (2.34 g, $14 \mathrm{mmol})$. After the completion of reaction (12h), filtered to remove solid $\mathbf{6}$ and extracted the aqueous layer on acidified with conc $\mathrm{HCl}$ to $\mathrm{pH}$ 2, Filter the yellow solid, suspended in hexane, filter and dry in 
vacuum to give trithiocarbonate $\mathbf{5 g}(1.22 \mathrm{~g}, 62 \%) . \mathrm{Mp}=186.59^{\circ} \mathrm{C} .{ }^{1} \mathrm{H}-\mathrm{NMR}(400 \mathrm{MHz}$, dmso$\left.\mathrm{d}_{6}\right):{ }^{\delta} 1.58\left(\mathrm{~s}, 12 \mathrm{H}, \mathrm{S}-\mathrm{C}\left(\mathrm{CH}_{3}\right)_{2}-\mathrm{COOH}\right) .{ }^{13} \mathrm{C}-\mathrm{NMR}\left(100.6 \mathrm{MHz}, \mathrm{dmso}^{\left.-\mathrm{d}_{6}\right):}{ }^{\delta} 173.9(\mathrm{C}=\mathrm{O}), 57.0\right.$ (S-C(CH$\left.)_{2}-\mathrm{COOH}\right), 25.7\left(\mathrm{~S}-\mathrm{C}\left(\mathrm{CH}_{3}\right)_{2}-\mathrm{COOH}\right) . \mathrm{IR}(\mathrm{KBr})$ (wavenumber, $\left.\mathrm{cm}^{-1}\right): 2985(\mathrm{C}-\mathrm{Cs})$, 1593, $1700(\mathrm{C}=\mathrm{O}), 1285,1063(\mathrm{C}=\mathrm{S}), 805$ and $761(\mathrm{C}-\mathrm{Cb})$. Elemental Analysis, Calcd for $\mathrm{C}_{9} \mathrm{H}_{14} \mathrm{O}_{4} \mathrm{~S}_{3}:$ C) $38.28 \%$, H) $5.00 \%$. Found: C) $38.71 \%$, H) $5.04 \%$.

Carbonotrithioic acid, bis(1-phenylethyl) $\operatorname{ester}^{34}$ (5h): In the manner described above, to a<smiles>CC(SC(=S)SC(C)c1ccccc1)c1ccccc1</smiles>
$6.45 \mathrm{~mL}(1.0 \mathrm{~g}, 3.6 \mathrm{mmol})$ in situ solution of $3-$ dithiocarboxysulfanyl-propane-1-sulfonicacid disodium salt was treated 1-bromo ethyl benzene $(0.67 \mathrm{~g}, 3.6 \mathrm{mmol})$. After completion of reaction (12 h), filtered to remove solid 6 and extracted with ether $50 \mathrm{~mL} \times 2$ ), and the organic layer was washed with water $(100 \mathrm{~mL})$, brine $(100 \mathrm{~mL})$, and then dried over anhydrous magnesium sulfate and filtered. The filtrate was concentrated give trithiocarbonate $\mathbf{5 h}(460 \mathrm{mg}, 80 \%) .{ }^{1} \mathrm{H}-\mathrm{NMR}$ $\left(400 \mathrm{MHz}, \mathrm{CDCl}_{3}\right):{ }^{\delta} 7.35(\mathrm{~m}, 5 \mathrm{H}, \mathrm{Ph}), 5.32\left(\mathrm{q}, 1 \mathrm{H}, J=7.8-\mathrm{SCH}\left(\mathrm{CH}_{3}\right), 1.73-1.54(\mathrm{~m}, 3 \mathrm{H},(\mathrm{S}-\right.$ $\mathrm{C}\left(\mathrm{CH}_{3}\right){ }^{13} \mathrm{C}-\mathrm{NMR}\left(100.6 \mathrm{MHz}, \mathrm{CDCl}_{3}\right):{ }^{\delta} 140.9,128.5,127.6,127.6,49.9\left(\mathrm{~S}-\mathrm{CH}\left(\mathrm{CH}_{3}\right), 21.5\right.$ $\left(\mathrm{S}-\mathrm{C}\left(\mathrm{CH}_{3}\right)\right.$.

Carbonotrithioic acid, bis[(4-bromophenyl) methyl] ester $^{35}$ (5i): In the manner described above, to a $6.45 \mathrm{~mL}(1.0 \mathrm{~g}, 3.6 \mathrm{mmol})$ 4-bromobenzylbromide $(0.67 \mathrm{~g}, 3.6 \mathrm{mmol})$. After the completion of reaction (12h), filtered to 
remove solid 6 and extracted the aqueous layer with ether $(50 \mathrm{~mL} \times 2)$, and the organic layer was washed with water $(100 \mathrm{~mL})$, brine $(100 \mathrm{~mL})$, and then dried over anhydrous magnesium sulfate and filtered. The filtrate was concentrated gives trithiocarbonate 5i $(560 \mathrm{mg}, 70 \%)$. Mp $=$ $93.27^{\circ} \mathrm{C}$. The compounds $\mathbf{5 i}$ is confirmed by single crystals data. ${ }^{1} \mathrm{H}-\mathrm{NMR}\left(400 \mathrm{MHz}, \mathrm{CDCl}_{3}\right):{ }^{\delta}$ $7.44(\mathrm{~d}, J=8.4 \mathrm{~Hz}, 2 \mathrm{H}), 7.21(\mathrm{~d}, J=8.4 \mathrm{~Hz}, 2 \mathrm{H}), 4.54$ (s, 2H, -SCHPh). ${ }^{13} \mathrm{C}-\mathrm{NMR}(100.6 \mathrm{MHz}$, $\left.\mathrm{CDCl}_{3}\right):{ }^{\delta} 134.0,131.7,130.6,121.7,40.6(\mathrm{~S}-\mathrm{CHPh}) . \mathrm{IR}(\mathrm{KBr}): 2920,1895,1402,720$ (medium), 1483, 1058, 1009, $795 \mathrm{~cm}^{-1}$ (strong). The impurity cyclic bromine is also confirmed by single crystals data.

Carbonotrithioic acid, bis[(4-methoxyphenyl) methyl] $\operatorname{ester}^{36}(\mathbf{5 j})$ : In the manner described<smiles>COc1ccc(CSC(=S)SCc2ccc(OC)cc2)cc1</smiles>
above, to a $6.45 \mathrm{~mL}(1.0 \mathrm{~g}, 3.6 \mathrm{mmol})$ situ solution of 3 dithiocarboxysulfanyl-propane-1sulfonicacid disodium salt was treated

4-methoxybenzylchloride (563 g, $3.6 \mathrm{mmol})$. After the completion of reaction (12h), filtered to remove solid 6 and extracted the aqueous layer with ether $(50 \mathrm{~mL} \times 2)$, and the organic layer was washed with water $(100 \mathrm{~mL})$, brine $(100 \mathrm{~mL})$, and then dried over anhydrous magnesium sulfate and filtered. The filtrate was concentrated gives trithiocarbonate $\mathbf{5 j}$ (490 $\mathrm{mg}, 78 \%$ ). ${ }^{1} \mathrm{H}-\mathrm{NMR}$ $\left(400 \mathrm{MHz}, \mathrm{CDCl}_{3}\right):{ }^{\delta} 7.26(\mathrm{~d}, J=8.6 \mathrm{~Hz}, 2 \mathrm{H}), 6.84(\mathrm{~d}, J=8.7 \mathrm{~Hz}, 2 \mathrm{H}), 4.57(\mathrm{~s}, 2 \mathrm{H},-\mathrm{SCHPh})$, $3.80\left(\mathrm{~s}, 3 \mathrm{H},\left(\mathrm{PhOCH}_{3}\right) .{ }^{13} \mathrm{C}-\mathrm{NMR}\left(100.6 \mathrm{MHz}, \mathrm{CDCl}_{3}\right):{ }^{\delta} 223.1(\mathrm{C}=\mathrm{S}), 159.1,130.5,126.6\right.$, 114.1, 55.2, 41.1.

Carbonotrithioic acid, bis[(4-chlorophenyl) methyl] $\operatorname{ester}^{37}$ (5k): In the manner described<smiles>S=C(SCc1ccc(Cl)cc1)SCc1ccc(Cl)cc1</smiles>
above, to a $6.45 \mathrm{~mL}(1.0 \mathrm{~g}, 3.6 \mathrm{mmol})$ situ solution of 3-dithiocarboxysulfanyl-propane-1-sulfonicacid 
disodium salt was treated 4-chlorobenzylchloride $(577 \mathrm{mg}, 3.6 \mathrm{mmol})$. After the completion of reaction (12 h), filtered to remove solid 6 and extracted the aqueous layer with ether (50 $\mathrm{mL} \times 2)$, and the organic layer was washed with water $(100 \mathrm{~mL})$, brine $(100 \mathrm{~mL})$, and then dried over anhydrous magnesium sulfate and filtered. The filtrate was concentrated gives trithiocarbonate 5k (515 g, 80\%). Melting point is $55-57^{\circ} \mathrm{C}{ }^{1} \mathrm{H}-\mathrm{NMR}\left(400 \mathrm{MHz}, \mathrm{cdcl}_{3}\right):{ }^{\delta} 7.25$ (s, 4H), 4.55 (s, 2H). ${ }^{13} \mathrm{C}-\mathrm{NMR}\left(400 \mathrm{MHz}, \mathrm{cdcl}_{3}\right):{ }^{\delta} 133.6,133.5,130.5,128.8,40.5 . \quad$ IR (KBr): 2922, 1894 , 1404, 722 (medium), 1489, 1091, 1057, $796 \mathrm{~cm}^{-1}$ (strong). The compounds $\mathbf{5 k}$ is confirmed by single crystals data.

bis[2-(2-methoxyethoxy)-ethyl]-disulfide (7a): To a $6.45 \mathrm{~mL}(1.0 \mathrm{~g}, 3.6 \mathrm{mmol})$ in situ solution

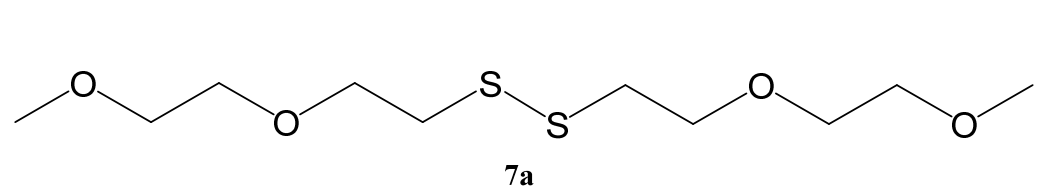

of 6, was added compounds 2a $(0.66 \mathrm{~g}, 3.6 \mathrm{mmol})$ and heat at $80^{\circ} \mathrm{C}$ for $2 \mathrm{~h}$. After completion of reaction dilute the reaction mixture with water $(50 \mathrm{~mL})$ and extract with ether $(25 \mathrm{~mL} \mathrm{X} \mathrm{2)}$. Wash the ether layer with wash $(50 \mathrm{~mL})$, brine solution $(50 \mathrm{~mL})$, dried over $\mathrm{MgSO}_{4}$ and filter. The filtrate was concentrated to obtain disulfide 7a as residual oil (202 mg, $42 \%$ ). ${ }^{1} \mathrm{H}-\mathrm{NMR}$ (400 MHz, $\left.\mathrm{cdcl}_{3}\right):{ }^{\delta} 3.74-3.61\left(\mathrm{~m}, 8 \mathrm{H}, \mathrm{CH}_{3} \mathrm{O}-\mathrm{CH}_{2}-\mathrm{CH}_{2} \mathrm{O}-\right)$, 3.55-3.53 (dd, 4H, $\mathrm{J}=2.5 \mathrm{~Hz}$ and $3.8 \mathrm{~Hz}$, $\left.-\mathrm{OCH}_{2} \mathrm{CH}_{2} \mathrm{~S}\right), 3.38\left(\mathrm{~s}, 6 \mathrm{H},-\mathrm{OCH} \boldsymbol{H}_{3}\right), 2.78-2.75\left(\mathrm{t}, 4 \mathrm{H}, \mathrm{J}=6.9 \mathrm{~Hz},-\mathrm{CH}_{2} \mathrm{~S}\right) \cdot{ }^{13} \mathrm{C}-\mathrm{NMR}(100.6 \mathrm{MHz}$, $\left.\mathrm{cdcl}_{3}\right):{ }^{\delta} 71.8\left(\mathrm{CH}_{3} \mathrm{O}-\mathrm{CH}_{2}-\mathrm{CH}_{2} \mathrm{O}-\right), 71.0\left(\mathrm{CH}_{3} \mathrm{O}-\mathrm{CH}_{2}-\mathrm{CH}_{2} \mathrm{O}-\right), 70.2\left(-\mathrm{OCH}_{2} \mathrm{CH}_{2} \mathrm{~S}\right), \quad 59.7 \quad(-$ $\left.\mathrm{OCH}_{3}\right), 31.7\left(-\mathrm{CH}_{2} \mathrm{~S}\right) . \quad \mathrm{IR}(\mathrm{KBr})$ (wavenumber, $\left.\mathrm{cm}^{-1}\right): 2876$ (C-Cs), 1354, 1275, 1198, 1100 $(\mathrm{C}=\mathrm{S}), 733(\mathrm{C}-\mathrm{Cb})$. Elemental Analysis, Calcd for $\mathrm{C}_{10} \mathrm{H}_{22} \mathrm{O}_{4} \mathrm{~S}_{2}$ : C) $44.42 \%$, H) $8.20 \%$. Found: C) $44.29 \%$, H) $8.19 \%$. 
bis[2-(2-methoxyethoxy)-ethanethiol ${ }^{27}$ (4a): The aqueous layer on acidified with concentrated

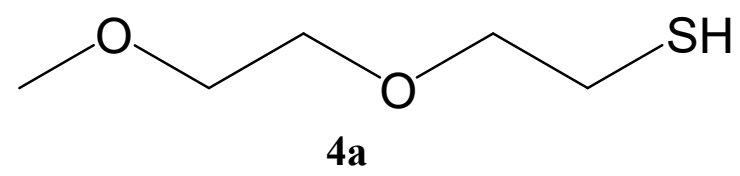
$\mathrm{HCl}$ to $\mathrm{pH}=2$, then extract with ether $(25 \mathrm{~mL} \mathrm{X} \mathrm{2)}$, wash the ether layer with water $(50 \mathrm{~mL})$, brine solution $(50 \mathrm{~mL})$, dried over $\mathrm{MgSO}_{4}$ and filter. The filtrate was concentrated to give thiol $\mathbf{4 a}$ as residual oil. (216 $\mathrm{mg}, 44 \%)$.

bis(1-phenylethyl) disulfide ${ }^{38}$ (7h): To a $6.45 \mathrm{~mL}(1.0 \mathrm{~g}, 3.6 \mathrm{mmol})$ in situ solution of $\mathbf{6}$, was<smiles>CC(SSC(C)c1ccccc1)c1ccccc1</smiles>
added 1-bromo ethyl benzene $(0.67 \mathrm{~g}, 3.6 \mathrm{mmol})$ and heat at $80^{\circ} \mathrm{C}$ for $2 \mathrm{~h}$. The reaction mixture was dilute with water and extract with ether $(25 \mathrm{~mL} \mathrm{X}$ 2 ), and the organic layer was washed with water $(100 \mathrm{~mL})$, brine $(100 \mathrm{~mL})$, and then dried over anhydrous magnesium sulfate and filtered. The filtrate was concentrated give disulfide $7 \mathbf{i}$ (195 mg, 40\%). NMR data is consistent with reported values.

1-phenylethyl thiol ${ }^{39}(\mathbf{4 h})$ : The aqueous layer on acidified with concentrated $\mathrm{HCl}$ to $\mathrm{pH}=2$, then<smiles>CC(S)c1ccccc1</smiles>
extract with ether $(25 \mathrm{~mL} \mathrm{X} \mathrm{2)}$, wash the ether layer with water $(50 \mathrm{~mL})$, brine solution $(50 \mathrm{~mL})$, dried over $\mathrm{MgSO}_{4}$ and filter. The filtrate was concentrated to give thiol $4 \mathbf{i}$ as residual oil. (170 $\mathrm{mg}, 36 \%)$. NMR data is consistent with reported values.

Bis(p-bromobenzyl) disulfide ${ }^{40}$ (7i): To a $6.45 \mathrm{~mL}(1.0 \mathrm{~g}, 3.6 \mathrm{mmol})$ in situ solution of 6, was<smiles>Brc1ccc(CSSCc2ccc(Br)cc2)cc1</smiles>
added 4-bromobenzylbromide $(0.9 \mathrm{~g}$, $3.6 \mathrm{mmol}$ ) and heat at $80^{\circ} \mathrm{C}$ for $2 \mathrm{~h}$. The reaction mixture was dilute with water 
and extract with ether (25 $\mathrm{mL} \mathrm{X} \mathrm{2),} \mathrm{and} \mathrm{the} \mathrm{organic} \mathrm{layer} \mathrm{was} \mathrm{washed} \mathrm{with} \mathrm{water} \mathrm{(100} \mathrm{mL})$, brine $(100 \mathrm{~mL})$, and then dried over anhydrous magnesium sulfate and filtered. The filtrate was concentrated give disulfide $7 \mathbf{i}(138 \mathrm{mg}, 38 \%)$. NMR data is consistent with reported values.

p-bromobenzyl thiol ${ }^{41}$ (4i): The aqueous layer on acidified with concentrated $\mathrm{HCl}$ to $\mathrm{pH}=2$,

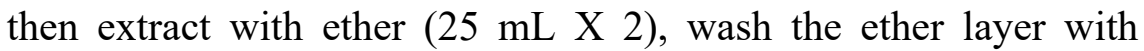<smiles>SCc1ccc(Br)cc1</smiles>

$4 \mathbf{i}$ water $(50 \mathrm{~mL})$, brine solution $(50 \mathrm{~mL})$, dried over $\mathrm{MgSO}_{4}$ and filter. The filtrate was concentrated to give thiol $\mathbf{4 i}$ as residual oil. (290 mg, 40\%). NMR data is consistent with reported values.

Bis(p-methoxybenzyl) disulfide ${ }^{42}(\mathbf{7 j}):$ To a $6.45 \mathrm{~mL}(1.0 \mathrm{~g}, 3.6 \mathrm{mmol})$ in situ solution of $\mathbf{6}$,<smiles>COc1ccc(CSSCc2ccc(OC)cc2)cc1</smiles>
h. The reaction mixture was dilute with water and extract with ether $(25 \mathrm{~mL} X 2)$, and the organic layer was washed with water $(100 \mathrm{~mL})$, brine $(100 \mathrm{~mL})$, and then dried over anhydrous magnesium sulfate and filtered. The filtrate was concentrated give disulfide $7 \mathbf{i}$ (200 mg, 36\%). NMR data is consistent with reported values.

p-methoxybenzyl thiol ${ }^{\mathbf{4 3}}(\mathbf{4 j})$ : The aqueous layer on acidified with concentrated $\mathrm{HCl}$ to $\mathrm{pH}=2$,<smiles>COc1ccc(CS)cc1</smiles>
then extract with ether ( $25 \mathrm{~mL} \mathrm{X} \mathrm{2),} \mathrm{wash} \mathrm{the} \mathrm{ether} \mathrm{layer} \mathrm{with}$ water $(50 \mathrm{~mL})$, brine solution $(50 \mathrm{~mL})$, dried over $\mathrm{MgSO}_{4}$ and filter. The filtrate was concentrated to give thiol $\mathbf{4 i}$ as residual 
oil. (210 mg, 38\%). NMR data is consistent with reported values.

Bis(p-chlorobenzyl) disulfide $\left.\mathbf{4 0}^{\mathbf{4 0}} \mathbf{7 k}\right)$ : To a $6.45 \mathrm{~mL}(1.0 \mathrm{~g}, 3.6 \mathrm{mmol})$ in situ solution of $\mathbf{6}$, was

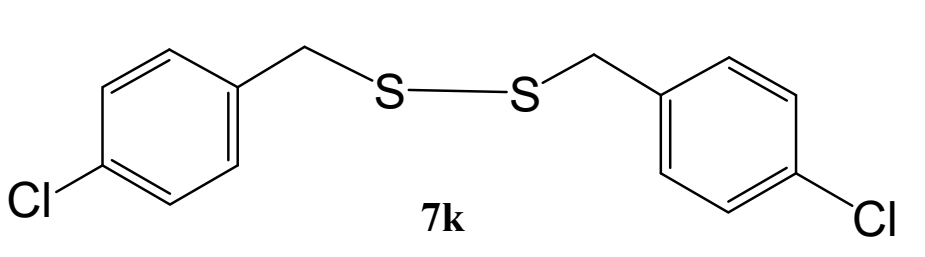
added treated with 4-chlorobenzyl chloride (580 mg, $0.0036 \mathrm{~mol})$ and heat at $80^{\circ} \mathrm{C}$ for $2 \mathrm{~h}$. The reaction mixture was dilute with water and extract with ether $(25 \mathrm{~mL} \mathrm{X} \mathrm{2),} \mathrm{and} \mathrm{the} \mathrm{organic} \mathrm{layer} \mathrm{was} \mathrm{washed} \mathrm{with}$ water $(100 \mathrm{~mL})$, brine $(100 \mathrm{~mL})$, and then dried over anhydrous magnesium sulfate and filtered. The filtrate was concentrated give $226 \mathrm{mg}$ of disulfide $7 \mathbf{k}$ in $40 \%$ yield. Melting point is 51 $52^{\circ} \mathrm{C} .{ }^{1} \mathrm{H}-\mathrm{NMR}\left(400 \mathrm{MHz}, \mathrm{cdcl}_{3}\right):{ }^{\delta}$ 7.30-7.28 (d, 2H, J = 8.3 Hz), 7.16-7.14 (d, 2H, J = 8.3 Hz), 3.57 (s, 2H). ${ }^{13} \mathrm{C}-\mathrm{NMR}\left(400 \mathrm{MHz}, \mathrm{cdcl}_{3}\right):{ }^{\delta}$ 135.8, 133.3, 130.6, 128.6, $42.4 \mathrm{IR}$ (KBr): 3044, 2907, 1901, 720(medium), 1487, 1092, 1012, $832 \mathrm{~cm}^{-1}$ (strong). The compounds $7 \mathbf{k}$ is confirmed by single crystals data.

p-chlorobenzyl thiol ${ }^{44}$ (4k): The aqueous layer on acidified with concentrated $\mathrm{HCl}$ to $\mathrm{pH}=2$, then extract with ether $(25 \mathrm{~mL}$ X 2), wash the ether layer with<smiles>SCc1ccc(Cl)cc1</smiles>

$4 \mathbf{k}$ water $(50 \mathrm{~mL})$, brine solution $(50 \mathrm{~mL})$, dried over $\mathrm{MgSO}_{4}$ and filter. The filtrate was concentrated to give thiol $\mathbf{4 k}$ as residual oil. (238 mg, 42\%). ${ }^{1} \mathrm{H}-\mathrm{NMR}\left(400 \mathrm{MHz}, \mathrm{cdcl}_{3}\right):{ }^{\delta} 7.25-7.19(\mathrm{~m}, 4 \mathrm{H})$

$3.66(\mathrm{~d}, 2 \mathrm{H}, J=8.3 \mathrm{~Hz}), 1.75(\mathrm{~s}, 1 \mathrm{H}, J=8.3 \mathrm{~Hz}) .{ }^{13} \mathrm{C}-\mathrm{NMR}\left(400 \mathrm{MHz}, \mathrm{cdcl}_{3}\right):{ }^{\delta} 139.4,132.6$, 129.2, 128.6 28.1. IR (KBr): 3047, 2933, 1997, (medium), 1490, 1264, 1092, $830 \mathrm{~cm}^{-1}$ (strong).

\section{Reference:}

1. Hawker, C. J.; Bosman, A. W.; Harth, E., New Polymer Synthesis by Nitroxide Mediated Living Radical Polymerizations. Chem. Rev. (Washington, D. C.) 2001, 101 (12), 3661-3688.

2. Haven, J. J.; Hendrikx, M.; Junkers, T.; Leenaers, P. J.; Tsompanoglou, T.; Boyer, C.; $\mathrm{Xu}$, J.; Postma, A.; Moad, G., Elements of RAFT navigation: RAFT 20 years later: RAFT- 
synthesis of uniform, sequence-defined (co)polymers. ACS Symp. Ser. 2018, 1284 (Reversible Deactivation Radical Polymerization: Mechanisms and Synthetic Methodologies), 77-103.

3. De, P.; Gondi, S. R.; Roy, D.; Sumerlin, B. S., Boronic Acid-Terminated Polymers: Synthesis by RAFT and Subsequent Supramolecular and Dynamic Covalent Self-Assembly. Macromolecules (Washington, DC, U. S.) 2009, 42 (15), 5614-5621.

4. Zard, S. Z., Discovery of RAFT/MADIX process: mechanistic insights and polymer chemistry implications. Macromolecules (Washington, DC, U. S.) 2020, 53 (19), 8144-8159.

5. Favier, A.; Charreyere, M.-T.; Chaumont, P.; Pichot, C., Study of the RAFT Polymerization of a Water-Soluble Bisubstituted Acrylamide Derivative. 1. Influence of the Dithioester Structure. Macromolecules 2002, 35 (22), 8271-8280.

6. Thomas, D. B.; Convertine, A. J.; Myrick, L. J.; Scales, C. W.; Smith, A. E.; Lowe, A. B.; Vasilieva, Y. A.; Ayres, N.; McCormick, C. L., Kinetics and Molecular Weight Control of the Polymerization of Acrylamide via RAFT. Macromolecules 2004, 37 (24), 8941-8950.

7. Lai, J. T.; Filla, D.; Shea, R., Functional Polymers from Novel Carboxyl-Terminated Trithiocarbonates as Highly Efficient RAFT Agents. Macromolecules 2002, 35 (18), 6754-6756.

8. Harrisson, S.; Wooley, K. L., Shell-crosslinked nanostructures from amphiphilic AB and ABA block copolymers of styrene-alt-(maleic anhydride) and styrene: polymerization, assembly and stabilization in one pot. Chem. Commun. (Cambridge, U. K.) 2005, (26), 3259-3261.

9. Llinas, A.; Ahmed, N.; Cordaro, M.; Laws, A. P.; Frere, J.-M.; Delmarcelle, M.; Silvaggi, N. R.; Kelly, J. A.; Page, M. I., Inactivation of Bacterial DD-Peptidase by $\beta$-Sultams. Biochemistry 2005, 44 (21), 7738-7746.

10. Voronkov, M. G.; Panova, G. M.; Timokhina, L. V.; Gromkova, R. A., Thermal Reactions of Dibenzyl Disulfide and Dibenzyl Sulfide with Metals: A New Route to transStilbene and Dibenzyl. Russ. J. Gen. Chem. 2004, 74 (7), 1043-1045.

11. El-Wassimy, M. T. M.; Joergensen, K. A.; Lawesson, S. O., The reaction of thiocarbonyl compounds, S,S-acetals and thiols with the 1,4-dioxane-dibromine complex. Convenient methods for the transformation of thiocarbonyl compounds and S,S-acetals into the corresponding carbonyl synthons, and the oxidation of thiols to the corresponding disulfides. Chem. Scr. 1984, 24 (2), 80-3.

12. Gastaldi, S.; Escoubet, S.; Vanthuyne, N.; Gil, G.; Bertrand, M. P., Dynamic Kinetic Resolution of Amines Involving Biocatalysis and in Situ Free Radical Mediated Racemization. Organic Letters 2007, 9 (5), 837-839.

13. Gondi, S. R.; Son, D. Y.; Biehl, E. R.; Vempati, R. K., Easy and Rapid Method for Disulfide Syntheses Using Nanophase-Manganese (VII) Oxide Coated Clay. Phosphorus, Sulfur, and Silicon and the Related Elements 2009, 185 (1), 34-39.

14. Relyea, D. I.; Tawney, P. O.; Williams, A. R., Reaction of the 2-Cyano-2-propyl Radical with Elemental Sulfur. The Journal of Organic Chemistry 1962, 27 (3), 1078-1079.

15. Martin, D. J.; Greco, C. C., Thiol synthesis. The Journal of Organic Chemistry 1968, 33 (3), 1275-1276.

16. Nishio, T., Direct conversion of alcohols into thiols. Journal of the Chemical Society, Perkin Transactions 1 1993, (10), 1113-1117.

17. Howard, J. L.; Schotten, C.; Alston, S. T.; Browne, D. L., Preparation of difluoromethylthioethers through difluoromethylation of disulfides using TMS-CF2H. Chemical Communications 2016, 52 (54), 8448-8451.

18. Ozturk, T.; Ertas, E.; Mert, O., Use of Lawesson's Reagent in Organic Syntheses. Chemical Reviews 2007, 107 (11), 5210-5278. 
19. Nath, U.; Chowdhury, D.; Pan, S. C., Nonenzymatic Dynamic Kinetic Resolution of in situ Generated Hemithioacetals: Access to 1,3-Disubstituted Phthalans. Advanced Synthesis \& Catalysis 2018, 360 (8), 1628-1633.

20. Glatzel, S.; Badi, N.; Päch, M.; Laschewsky, A.; Lutz, J.-F., Well-defined synthetic polymers with a protein-like gelation behavior in water. Chem Commun (Camb) 2010, 46 (25), 4517-4519.

21. Barbero, M.; Cadamuro, S.; Degani, I.; Dughera, S.; Fochi, R. Method for the preparation of mono- and bis-alkyl or aryl sulfonyl chlorides. IT91MI0882A1, 1992.

22. Zhang, H.; Gondi, S. R.; Son, D. Y., Poly[bis $(\mu 3-$ benzyloxyiminoacetato)nitratotrisilver(I)]. Acta Crystallogr., Sect. E: Struct. Rep. Online 2006, 62 (7), m1613-m1615.

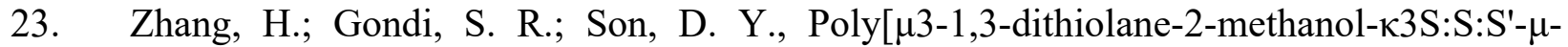
nitrato-silver(I)]. Acta Crystallogr., Sect. E: Struct. Rep. Online 2006, 62 (11), m3086-m3088.

24. Gondi, S. R.; Zhang, H.; Son, D. Y., Metallopolymer formation from the interaction of a hydrolyzed dithiolane-carboxylate ligand with Ag(I). J. Sulfur Chem. 2011, 32 (1), 17-21.

25. Benaglia, M.; Spisni, E.; Alberti, A.; Giorgini, L.; Magnoni, F.; Papi, A.; Treossi, E.; Palermo, V., Polymeric micelles using pseudo-amphiphilic block copolymers. Macromol. Symp. 2012, 313-314 (Functional Polymeric Materials and Composites), 51-58.

26. Glatzel, S.; Laschewsky, A.; Lutz, J.-F., Well-Defined Uncharged Polymers with a Sharp UCST in Water and in Physiological Milieu. Macromolecules 2011, 44 (2), 413-415.

27. Comasseto, J. V.; Guarezemini, A. S., Product class 5: alkanethiols. Sci. Synth. 2007, 39, 391-411.

28. Rucareanu, S.; Gandubert, V. J.; Lennox, R. B., 4-(N,N-Dimethylamino)pyridineProtected Au Nanoparticles: Versatile Precursors for Water- and Organic-Soluble Gold Nanoparticles. Chem. Mater. 2006, 18 (19), 4674-4680.

29. Lee, M. D.; Tong, W. Y.; Nebl, T.; Pearce, L. A.; Pham, T. M.; Golbaz-Hagh, A.; Puttick, S.; Rose, S.; Adams, T. E.; Williams, C. C., Dual Site-Specific Labeling of an Antibody Fragment through Sortase A and $\pi$-Clamp Conjugation. Bioconjugate Chem. 2019, 30 (10), 2539-2543.

30. Yamazaki, H.; Harada, H.; Matsuzaki, K.; Yoshioka, K.; Takase, M.; Ohki, E., Studies on bi-heterocyclic compounds. I. 6-Substituted dihydro-1,4-thiazinones. Chem. Pharm. Bull. 1987, 35 (6), 2243-53.

31. Stamenovic, M. M.; Espeel, P.; Van Camp, W.; Du Prez, F. E., Norbornenyl-Based RAFT Agents for the Preparation of Functional Polymers via Thiol-Ene Chemistry. Macromolecules (Washington, DC, U. S.) 2011, 44 (14), 5619-5630.

32. Campbell, B.; Hood, T.; Shank, N., Synthesis of a new disulfide Fmoc monomer for creating biologically susceptible linkages in peptide nucleic acid oligomers. Bioorg. Chem. 2019, 84, 394-398.

33. Grover, G. N.; Lee, J.; Matsumoto, N. M.; Maynard, H. D., Aminooxy and Pyridyl Disulfide Telechelic Poly(poly(ethylene glycol) acrylate) by RAFT Polymerization. Macromolecules (Washington, DC, U. S.) 2012, 45 (12), 4958-4965.

34. Yousefi, A., Superbasic system $\mathrm{CsOH} / \mathrm{DMSO}$ as a reagent for a fast one-step synthesis of symmetrical dialkyl trithiocarbonates. J. Sulfur Chem. 2015, 36 (6), 672-677.

35. Kiasat, A. R.; Mehrjardi, M. F., A novel one-step synthesis of symmetrical dialkyl trithiocarbonates in the presence of phase-transfer catalysis. J. Chin. Chem. Soc. (Taipei, Taiwan) 2008, 55 (3), 639-642. 
36. Harano, K.; Taguchi, T., Rearrangements and trans eliminations contrary to the Chugaev reaction. 12. Transformation of sodium O-benzyl xanthogenate to the corresponding trithiocarbonate. Yakugaku Zasshi 1974, 94 (12), 1495-502.

37. Aoyagi, N.; Endo, T., Functional RAFT agents for radical-controlled polymerization: Quantitative synthesis of trithiocarbonates containing functional groups as RAFT agents using equivalent amount of CS2. J. Polym. Sci., Part A: Polym. Chem. 2009, 47 (14), 3702-3709.

38. Antonietti, M.; Savatieiev, O.; Kurpil, B.; Dontsova, D. Photocatalytic system and applications. WO2019081036A1, 2019.

39. Knoppe, S.; Kothalawala, N.; Jupally, V. R.; Dass, A.; Buergi, T., Ligand dependence of the synthetic approach and chiroptical properties of a magic cluster protected with a bicyclic chiral thiolate. Chem. Commun. (Cambridge, U. K.) 2012, 48 (38), 4630-4632.

40. Abbasi, M.; Nowrouzi, N.; Mousavi, S., Aerobic Oxidation of Thiols and In Situ Generated Thiols to Symmetrical Disulfides (Disulfanes) Catalyzed by Na2S4O6. ChemistrySelect 2019, 4 (42), 12227-12231.

41. Bandgar, B. P.; Sadavarte, V. S., One pot rapid synthesis of thiols from alcohols under mild conditions. Synlett 2000, (6), 908-910.

42. Fritze, U. F.; von Delius, M., Dynamic disulfide metathesis induced by ultrasound. Chem. Commun. (Cambridge, U. K.) 2016, 52 (38), 6363-6366.

43. Sorribes, I.; Corma, A., Nanolayered cobalt-molybdenum sulphides (Co-Mo-S) catalyse borrowing hydrogen C-S bond formation reactions of thiols or H2S with alcohols. Chem. Sci. 2019, 10 (10), 3130-3142.

44. Eriksen, K.; Ulfkjaer, A.; Soelling, T. I.; Pittelkow, M., Benzylic Thio and Seleno Newman-Kwart Rearrangements. J. Org. Chem. 2018, 83 (18), 10786-10797. 